\title{
Design characteristics, primary stability and risk of fracture of orthodontic mini-implants: Pilot scan electron microscope and mechanical studies
}

\author{
André Walter ${ }^{1}$, Heinz Winsauer ${ }^{2}$, Jordi Marcé-Nogué ${ }^{3}$, Sergi Mojal ${ }^{4}$, Andreu Puigdollers ${ }^{5}$ \\ ${ }^{1}$ Associate professor, Universitat Internacional de Catalunya. School of Dentistry, Department of Orthodontics, PhD candidate, \\ Barcelona, Spain \\ ${ }^{2}$ Private practice, Bregenz, Austria \\ ${ }^{3}$ Researcher and lecturer. Departament de Resistència de Materials i Estructures a l'Enginyeria, Universitat Politècnica de \\ Catalunya, BarcelonaTech, Terrasa, Spain \\ ${ }^{4}$ Instituto municipal de Investigaciones Médicas (IMIM), Statistical analysis and research, Barcelona, Spain \\ ${ }^{5}$ Chairman and professor, Departament of Orthodontics, School of Dentistry, Universitat Internacional de Catalunya, Barcelona, \\ Spain
}

Correspondence:

Universitat Internacional de Catalunya

Campus Sant Cugat

Josep Trueta $s / n, 08195$

St. Cugat del Vallès,

Barcelona, Spain

apuigdollersp@gmail.com

Walter A, Winsauer H, Marcé-Nogué J, Mojal S, Puigdollers A. Design characteristics, primary stability and risk of fracture of orthodontic miniimplants: Pilot scan electron microscope and mechanical studies. Med Oral Patol Oral Cir Bucal. 2013 Sep 1;18 (5):e804-10.

http://www.medicinaoral.com/medoralfree01/v18i5/medoralv18i5p804.pdf

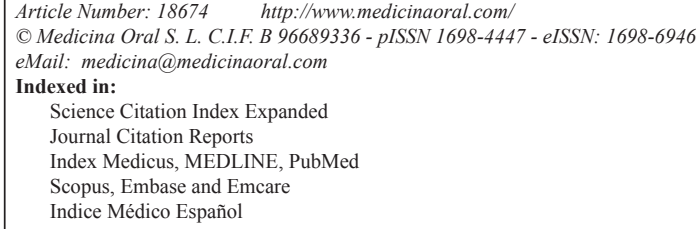

\begin{abstract}
Objectives: Orthodontic mini-implants (OMIs) are increasingly used in orthodontics but can fail for various reasons. This study investigates the effects of OMI design characteristics on the mechanical properties in artificial bone.

Material and Methods: Twelve self-drilling OMIs (2 small, 6 medium, 4 large) from 8 manufacturers were tested for their primary stability in simulated medium-high cancellous bone and the risk to fracture in high-density methacrylate blocks. For the assessments of the maximum insertion torque (IT) and torsional fracture (TF) 5 of each OMI were used and for the pull-out strength (POS) 10. The OMIs were inserted with a torque screwdriver $\left(12 \mathrm{sec} / 360^{\circ}\right)$ until the bottom at $8 \mathrm{~mm}$ depth was reached. OMI designs were analyzed with a scan electron microscope (SEM).

Results: SEM images revealed a great variation in product refinement. In the whole sample, a cylindrical OMI shape was associated with higher POS $(p<0.001)$ but lower IT $(p=0.002)$ values. The outer and inner OMI diameters were design characteristics well correlated with POS, IT and TF values (ranging from 0.601 to 0.961 ). Greater thread depth was related to greater POS values $(\mathrm{r}=0.628)$, although OMIs with similar POS values may have different IT values. Thread depth and pitch had some impact on POS. TF depended mainly on the OMI inner $(r=0.961)$ and outer diameters $(r=0.892)$. A thread depth to outer diameter ratio close to $40 \%$ increased TF risk.
\end{abstract}


Conclusions: Although at the same insertion depth the OMI outer and inner diameters are the most important factors for primary stability, other OMI design characteristics (cylindrical vs. conical, thread design) may significantly affect primary stability and torsional fracture. This needs to be considered when selecting the appropriate OMI for the desired orthodontic procedures.

Key words: Orthodontic mini-implants, primary stability, insertion torque, pullout strength, torsional fracture.

\section{Introduction}

The primary implant stability of orthodontic mini-implants (OMIs) is not only affected by the bone quality (ratio compact to trabecular bone) (1), and bone quantity (mineral density) (2), but also by the OMI design characteristics. The latter include length, diameter, thread depth, width, helix angle and pitch (axial distance between threads) variables, thread depth-to-outer diameter ratio, presence of flutes (recessed areas in the cross-sectional area of the OMI that carry bone chips away from the cutting edge as the screw rotates) and body shape (conical, cylindrical). Length and diameter are most important for the OMI primary stability $(3,4)$. Other OMI characteristics are in general not declared by the manufacturers.
The aim of the study was to assess the design characteristics of 12 commercially available self-drilling OMIs using a scanning electron microscope (SEM). Established test procedures were used to investigate the mechanical properties of the OMIs in terms of pullout strength (POS), insertion torques (IT) and, torsional fracture (TF).

\section{Material and Methods}

240 self-drilling titanium (Ti)-alloy (Ti-6AI-4Va) OMIs (12 types from 8 manufacturers) were investigated (Table 1, Fig. 1). Prior to the mechanical tests, pitch (axial distance between threads), thread (major diameter D2), shank (minor diameter D1) and thread angle were measured by means of SEM imaging (Quantas 200, EEUU).

Table 1. Diameter groups, diameters, lengths, type and manufacturer of the investigated OMIs.

\begin{tabular}{|l|c|c|c|l|l|}
\hline Group & $\mathbf{N}^{\mathbf{0}}$ & $\begin{array}{c}\text { Diameter } \\
\mathbf{m m}\end{array}$ & $\begin{array}{c}\text { Length } \\
\mathbf{m m}\end{array}$ & \multicolumn{1}{|c|}{ Type } & \multicolumn{1}{|c|}{ Manufacturer } \\
\hline small & 1 & 1.2 & 10 & Abso Anchor ${ }^{\circledR}$ & SH 1211-10 Dentos Daegu, Korea \\
& 2 & 1.3 & 11 & MAS Microbite Micerium & S.p.A Avegno, Italy \\
\hline medium & 3 & 1.5 & 11 & MAS Microbite Micerium & S.p.A Avegno, Italy \\
& 4 & 1.5 & 11 & Mondeal-PSM & PSM Tuttlingen, Germany \\
& 5 & 1.5 & 10 & Tekka CV H2 & Brignais, France \\
& 6 & 1.5 & 11 & Spider Screw ${ }^{\circledR}$ K1,HDC & Sarcedo, Italy \\
& 7 & 1.6 & 10 & Tomas ${ }^{\circledR}$-pin SD-N 10 & Dentaurum Inspringen, Germany \\
& 8 & 1.6 & 10 & Dual-Top TMJeil & Seoul, Korea \\
\hline large & 9 & 1.8 & 10 & 3M Unitek ${ }^{\text {TM }}$ & St Paul, MN, USA \\
& 10 & 2.0 & 10 & Dual-Top TMJeil & Seoul, Korea \\
& 11 & 2.0 & 11 & Tekka CV H2 & Brignais, France \\
& 12 & 2.0 & 11 & Mondeal-PSM & PSM Tuttlingen, Germany \\
\hline
\end{tabular}

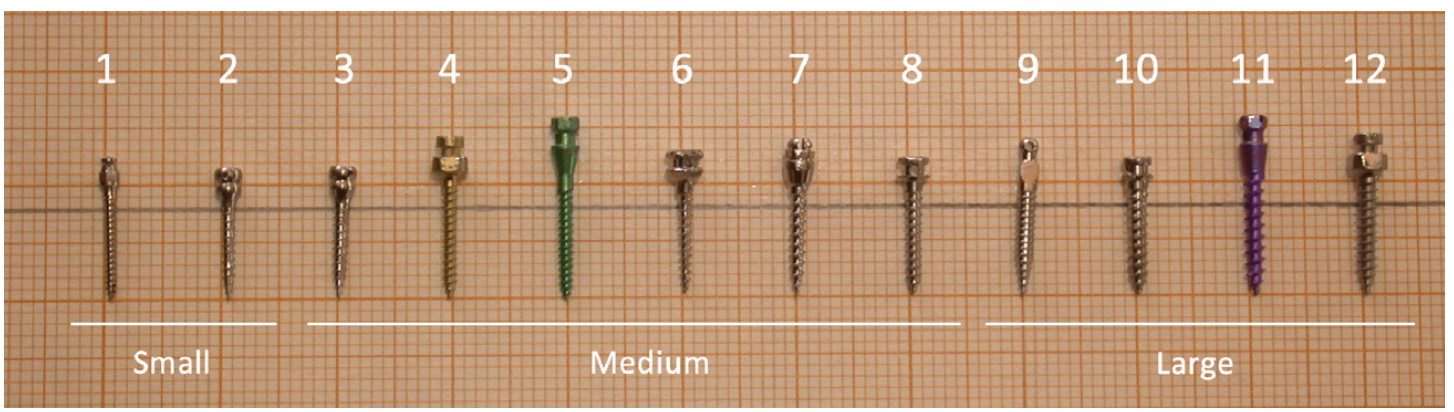

Fig. 1. The 12 different OMIs that were investigated in this study. 
Shank analysis was measured at 25-30x magnification, and thread details at $120 \mathrm{x}$ to $140 \mathrm{x}$ magnification.

The mechanical tests (POS, IT) were performed as described by others (5-7) using artificial bone simulating high cancellous bone (Sawbone $^{\mathrm{TM}}$ Pacific Reasearch Laboratories Inc., EEUU), 120 x 30 x 30mm with a density of 25 pounds per cubic foot; $\mathrm{g} / \mathrm{cc}=0.40$, compression (strength 12.9 Mpa, Modulus $317 \mathrm{Mpa}$ ), tension (strength 8.8 Mpa, Modulus 39.9 Mpa) and shear (strength 5.9 Mpa, Modulus 6.8 Mpa) (8). POS and IT were measured by inserting OMIs at $90^{\circ}$ into the artificial bone (9) without pre-drilling to a depth of $8 \mathrm{~mm}$ (accounting for gingival and bony anchorage support of $3 \mathrm{~mm}$ and $5 \mathrm{~mm}$, respectively) at a speed of $12 \mathrm{sec} / 360$. POS tests (10 OMIs of each brand per block) were performed with net axial forces using a Galdabini 1890 servo-hydraulic universal testing machine, Italy. Screw heads were clamped with a custom-made grip. A crosshead speed of $1.0 \mathrm{~mm} / \mathrm{min}$. was applied to extract the screws.

In order to measure torque (the clockwise torque resistance IT), screws were inserted perpendicularly (5 OMIs of each brand per block). Torque ( $\mathrm{Ncm}$ ) was measured with the digital torque tester Mecmesin AFT (0 to 500 Ncm, England). Methacrylate blocks (120x90x25) with greater IT resistance were used for the TF tests in order to simulate a high-dense cortical bone thickness (7). TF measurements (5 OMIs per block) were assessed after pre-drilling to a depth of $8 \mathrm{~mm}$ (drill diameter $0.5-1$ $\mathrm{mm}$ less than the individual OMI's diameters). Screws were inserted into the pilot hole and tightened $(3 \mathrm{sec}$./ 900) until they fractured.

One-Way ANOVA with Dunnett's T3 multiple comparison correction were used to detect differences in POS, IT and TF between OMI brands. Spearman's rho correlation coefficient were calculated to assess relationship between POS, IT, TF and the numerical OMI characteristics. Unpaired Student's T test was used to compare POS, IT and TF between shank type (cylindrical vs. conical). P values $<0.05$ were considered as statistically significant. All analysis were performed with SPSS 15.0 (SPSS Inc, Chicago, IL).

\section{Results}

OMIs were grouped into small, median and large according to their diameters. Their design characteristics are summarized in table 2. SEM magnifications of the threads show a great variation in product refinements, especially in the medium OMI group (Fig. 2).

In general, POS values depended on the OMI diameter. However, POS of the smallest OMI (No.1) was close to that of the median group. IT values were either below or around $10 \mathrm{Ncm}$ (Nos. 1,2,4,5, and 6) or higher than 10 Ncm (Nos. 3,7,8,9,10,11, and 12). OMIs Nos. 1, 2 and 6 fractured by a force between 8 to $16 \mathrm{Ncm}$, while the others fractured by a force between 22 to $48 \mathrm{Ncm}$ (Fig. 3).
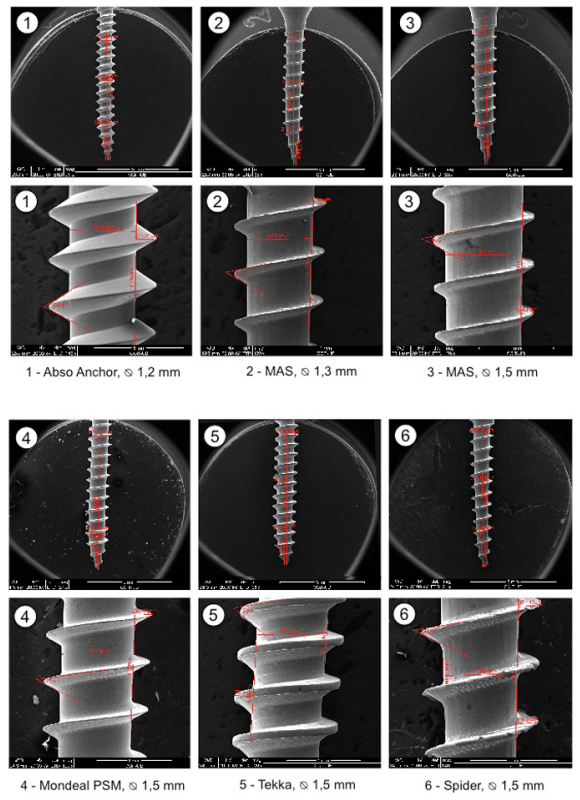

Fig. 2. SEM magnifications of the shanks (25-30x) and threads (120-140x) of the 12 different OMIs.

Table 2. Characteristics and SEM measurements regarding design, length, shape and thread of the 12 different OMIs tested.

\begin{tabular}{|c|c|c|c|c|c|c|c|c|c|c|c|}
\hline \multirow[b]{2}{*}{ OMI $\mathbf{N}^{\circ}$} & \multirow[b]{2}{*}{ Pitch mm } & \multicolumn{3}{|c|}{ Thread } & \multicolumn{3}{|c|}{ Lengths (mm) } & \multicolumn{2}{|c|}{ Diameters (mm) } & \multirow{2}{*}{$\begin{array}{c}\text { D2- } \\
\text { D1/D2x100 } \\
\text { ratio (\%) } \\
\end{array}$} & \multirow{2}{*}{$\begin{array}{c}\text { Conicity (2-8 } \\
\text { mm) } \%\end{array}$} \\
\hline & & Depth mm & $\begin{array}{c}\text { Angle } \\
\text { degress }\end{array}$ & $\begin{array}{l}N^{0} \text { at } \\
8 \mathrm{~mm}\end{array}$ & L1 & L2 & $\mathbf{L 3}$ & D1 & D2 & & \\
\hline 1 & 0.5 & 0.24 & 60 & 16 & 12.53 & 11.25 & 9.00 & 0.73 & 1.21 & $40 \%$ & $0.75 \%$ \\
\hline 2 & 0.6 & 0.19 & 40 & 14 & 11.60 & 11.30 & 8.00 & 0.87 & 1.25 & $30 \%$ & $2.83 \%$ \\
\hline 3 & 0.6 & 0.18 & 40 & 14 & 11.60 & 9.30 & 6.00 & 1.02 & 1.38 & $26 \%$ & $5.15 \%$ \\
\hline 4 & 0.7 & 0.24 & 35 & 11 & 13.82 & 12.50 & 10.00 & 0.96 & 1.44 & $33 \%$ & $0.16 \%$ \\
\hline 5 & 0.625 & 0.25 & 50 & 13 & 16.00 & 14.15 & 11.00 & 0.92 & 1.42 & $35 \%$ & $0.6 \%$ \\
\hline 6 & 0.7 & 0.27 & 35 & 11 & 12.60 & 12.10 & 9.00 & 0.89 & 1.43 & $38 \%$ & $6.1 \%$ \\
\hline 7 & 1.0 & 0.27 & 40 & 8 & 14.11 & 12.50 & 10.00 & 1.08 & 1.62 & $33 \%$ & $4.13 \%$ \\
\hline 8 & 0.7 & 0.235 & 50 & 11 & 12.20 & 10.75 & 9.00 & 1.10 & 1.57 & $30 \%$ & $1.1 \%$ \\
\hline 9 & 0.6 & 0.12 & 45 & 14 & 14.10 & 13.50 & 10.00 & 1.48 & 1.69 & $20 \%$ & $10 \%$ \\
\hline 10 & 0.7 & 0.30 & 50 & 11 & 12.30 & 10.75 & 9.00 & 1.34 & 1.94 & $30 \%$ & $1.3 \%$ \\
\hline 11 & 1.0 & 0.385 & 50 & 8 & 15.25 & 13.40 & 11.00 & 1.12 & 1.87 & $40 \%$ & $0.6 \%$ \\
\hline 12 & 0.7 & 0.32 & 35 & 11 & 14.31 & 13.40 & 10.00 & 1.30 & 1.94 & $35 \%$ & $0.7 \%$ \\
\hline
\end{tabular}



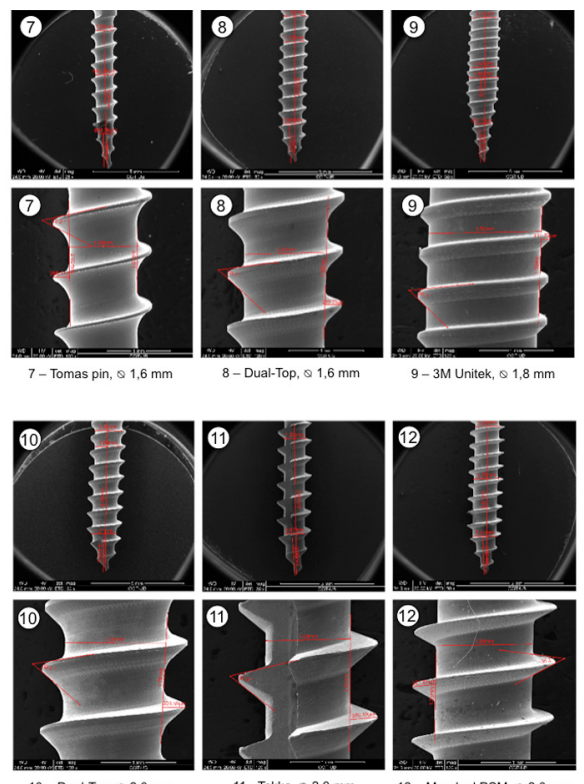

11 - Tekka, \& 2,0 mm

Fig. 3. Mechanical test results (mean $\pm \mathrm{SD}$ ) of the pull-out strength, insertion torque and torsional fracture of the 12 different OMIs.

In the whole sample, POS values depended on the OMI outer diameter (D2, $\mathrm{r}=0.846)$ and IT with the shank (D1, $\mathrm{r}=0.811$ ) (Table 3 ). POS values were higher in cylindrical OMIs $(p<0.001)$, whereas IT values were higher in the conical ones $(p<0.002)$. TF values correlated highly with the screw shank (D1, r=0.961) and the outer diameter (D2, $r=0.892)$. Statistical signification between pitch and thread depth with POS was high $(\mathrm{p}<0.001)$ but the intensity of the correlation of pitch and thread depth with POS was in fact low ( $\mathrm{r}=0.54$ and $\mathrm{r}=0.628$, respectively). POS was best correlated in the whole sample with the major diameter (D2) and IT with the shank (D1). TF showed levels of correlation with both D1 and D2. The diameters (D1 and D2) showed moderate to high correlations with all mechanical test results. Within the medium diameter OMI group, conical screws had higher IT $(\mathrm{p}<0.001)$ and TF $(\mathrm{p}=0.015)$ values than cylindrical OMIs, although no statistical significant differences in POS has been seen. The greater the angle, the greater was IT $(p=0.002)$ and the greater the pitch, the greater was POS $(\mathrm{p}<0.001)$, but correlations were moderate $(\rho=0.534$ and $\rho=0.637$, respectively).

Within the large diameter OMI group, conical screws had higher IT values and lower POS values than cylindrical OMIs. In this small group also a trend towards higher TF values was noted for the conical OMIs, but in the limit of the statistical signification. $(\mathrm{p}=0.049)$. However, the results need to confirmed in a greater sample. The greater the angle, the greater was IT $(p<0.001)$, but the correlation was only moderate $(\rho=0.686)$. The greater the pitch and the thread depth, the higher was TF (negative correlation) and POS but correlations were moderate to poor being $\rho=-0.732$ and $\rho=-0.622$ for TF and $\rho$ $=0.524$ and $\rho=0.688$ for POS, respectively (Table 3 ). This indicates that OMIs with lower pitch and thread depth are less easy to fracture. Pearson correlations between the results of the different tests of the medium OMI group did not reveal any relation between POS and IT $(r=0.054$ $, p=0.774)$.

\section{Discussion}

Our SEM results show that the OMI production goes along with different refinements. The importance of less accurate production is not yet known but it may well be that metallic detachments may enter the bone

Table 3. Correlations of total, medium and large OMI design characteristic and mechanical tests.

\begin{tabular}{|c|c|c|c|c|c|c|c|c|c|c|}
\hline & \multicolumn{3}{|c|}{12 OMIs types } & \multicolumn{3}{|c|}{ medium OMIs } & \multicolumn{3}{|c|}{ large OMIs } \\
\hline & & POS & IT & TF & POS & IT & TF & POS & IT & TF \\
\hline \multicolumn{11}{|l|}{ Shape $(* *)$} \\
\hline Cylindrical & Mean (SD) & $291(72.1)$ & $10.0(3.88)$ & $26.5(13.6)$ & $246(18.4)$ & $7.76(2.1)$ & $21.3(1.8)$ & $373(32.8)$ & $14.3(1.1)$ & $41.0(5.8)$ \\
\hline \multirow[t]{2}{*}{ Conical $(>1 \%)$} & Mean (SD) & $245(47,6)$ & $12.7(7.43)$ & $29.4(12.0)$ & $244(31.9)$ & $11.5(2.1)$ & $24.9(5.6)$ & $287(28.1)$ & $16.1(1.3)$ & $45.7(4.0)$ \\
\hline & p-value & $<0.001$ & 0.002 & 0.384 & 0.731 & $<0.001$ & 0.015 & $<0.001$ & 0.002 & 0.049 \\
\hline \multirow[t]{2}{*}{ Angle (*) } & $?$ & 0.003 & 0.08 & -0.108 & 0.222 & 0.534 & 0.401 & -0.364 & 0.686 & -0.351 \\
\hline & p-value & 0.973 & 0.546 & 0.412 & 0.089 & 0.002 & 0.028 & 0.021 & 0.001 & 0.129 \\
\hline \multirow[t]{2}{*}{ Pitch (*) } & $?$ & 0.54 & 0.282 & 0.312 & 0.637 & 0.024 & 0.333 & 0.524 & -0.080 & -0.732 \\
\hline & p-value & $<0.001$ & 0.029 & 0.015 & $<0.001$ & 0.902 & 0.072 & 0.001 & 0.736 & $<0.001$ \\
\hline \multirow[t]{2}{*}{ Thread depth (*) } & $?$ & 0.628 & 0.117 & 0.159 & 0.295 & -0.453 & -0.329 & 0.688 & -0.144 & -0.622 \\
\hline & p-value & $<0.001$ & 0.373 & 0.225 & 0.022 & 0.012 & 0.075 & $<0.001$ & 0.544 & 0.003 \\
\hline \multirow[t]{2}{*}{ Shank (D1) (*) } & $?$ & 0.601 & 0.811 & 0.961 & 0.45 & 0.671 & 0.917 & -0.662 & 0.176 & 0.811 \\
\hline & p-value & $<0.001$ & $<0.001$ & $<0.001$ & $<0.001$ & $<0.001$ & $<0.001$ & $<0.001$ & 0.457 & $<0.001$ \\
\hline Major (D2) (*) & $?$ & 0.846 & 0.743 & 0.892 & 0.61 & 0.288 & 0.596 & 0.535 & -0.060 & -0.304 \\
\hline Diameter & p-value & $<0.001$ & $<0.001$ & $<0.001$ & $<0.001$ & 0.123 & 0.001 & $<0.001$ & 0.802 & 0.193 \\
\hline
\end{tabular}

(*) Spearman's correlation coefficient.

(**) Student's T test.

POS (Pullout Strength), IT (Insertion Torque), TF (Torsional Fracture); $\mathrm{p}<0.05$ is considered as significant. 
(Fig. 2, No 6). The mechanical test results confirm that the overall OMI design has an impact on the mechanical properties. Manufacturers should therefore provide more information on OMIs than solely their lengths and outer diameters so that orthodontists can select the appropriate OMI for the desired procedure. There is no doubt that an increase in outer diameter and length will increase the primary stability of OMIs (Fig. 3) (4). Accordingly, the fracture resistance index increased for each $0.1 \mathrm{~mm}$ added in outer diameter (10). Our data are compatible with this finding by showing a tendency towards increased fracture risk in small, medium and large OMIs when the thread depth to outer diameter ratio reaches about $40 \%$ (reflecting a shank loss of 0.1 or $0.3 \mathrm{~mm}$ ) (Fig. 3, Nos. 1,6,11). In agreement with the pertinent literature, conical OMIs had higher IT values (11) and slightly lower POS values (5) than cylindrical OMIs. The higher IT values of the taper shape are in general associated with higher compression forces during placement (12) which may result in necrosis of osteocytes and bone resorption. In case of thick cortical bone, pre-drilling may reduce microdamage without affecting the OMI stability (13). By contrast, the use of a narrow drill produced significant bone damage (14). Twelve weeks after OMI placement, the initial difference in primary stability between cylindrical and conical OMIs was no longer seen (15). This indicates that only at the beginning of the orthodontic treatment the OMI shape design is of importance.

Under- or oversized thread pitches being less appropriate for clinical use (1). However, a double thread with decreasing pitch in the neck may counteract this disadvantage by increasing stability due to enhanced interlock surface between OMI and bone (16). An increase in thread angle increased IT. A decrease in thread depth decreased the risk to fracture in the large OMIs group $(\mathrm{p}=0.003, \mathrm{r}=-0.622)$, with low-moderate correlation (17). This phenomenon is only seen in the large group where differences among pitch and thread depth between specimens are greater than in the median group, a more homogeneous group (Tables 2,3). However, deeper thread depths may increase stabilization in poorer bone quality situations. Large diameter OMIs had higher IT and POS values than smaller OMIs (Fig. 3).

-Small Diameter OMIs: Small diameter OMIs are suitable for narrow interradicular placement as required for simple tooth movements in the maxilla or mandible. OMIs with a small pitch are easier to insert. OMIs with less than $8 \mathrm{~mm}$ in length and $1.2 \mathrm{~mm}$ in diameter should be avoided due to a high failure rate (18). A high thread depth to outer diameter ratio providing a thinner shank is more prone to $\mathrm{TF}$ (Fig. 3, No 1) and less appropriate for clinical use. Immediate loading of small OMIs up to $20 \mathrm{~N}$ is possible (18).

Our results confirm that the cylindrical OMI Nol had lower IT values than the conical OMI No 2 (11). Small self-drilling OMIs are not suitable to be inserted into bone with a density greater than 40 pounds per cubic foot (19). The TF value was about half for the cylindrical than the conical (Fig. 3). The force that might fracture the OMI was close to the placement torque indicating that the OMI might already fracture during insertion. The greater thread depth of OMI Nol (ratio 40\%) (Table 2) correspond to the higher POS (Fig. 3) which is favourable in principle, but may not outweigh the increased fracture risk disadvantages. OMI No 2 could therefore be preferable to OMI No 1 for simple tooth movements due to its better characteristics against torsional fracture during insertion.

-Medium Diameter OMIs: Medium diameter OMIs are needed for en masse teeth movement or intrusion of teeth. The greater the length and diameter of the medium OMI, the higher was the implant stability with increasing forces (9). No statistical differences was observed in POS between conical and cylindrical OMIs, but the conical shape were superior in primary stability in terms of IT (Table 3). OMIs with low thread angles and sharp threads (Fig. 3, No 4) are probably more appropriate for denser bone, e.g. the mandible or the palate due to a lower IT and risk of fracture (Fig. 3). Our results suggest that OMIs the thread depth to outer diameter ratio should not exceed $33 \%$ (1 to 3 ) in order to lower TF risk when OMIs are placed in dense bone. Lower IT values were associated with a trend towards less inflammation in the local bone tissue around the OMIs (20).

Motoyoshi and co-workers $(21,22)$ evaluated a range of 5-10 $\mathrm{Ncm}$ as optimum IT for pre-drilling OMIs in both, the maxilla and mandible. For self-drilling OMIs, the adequate IT may be somewhat higher (20). Using conical OMIs, especially Nos. 3,7 and 8, a pilot drill is recommended to decrease the IT for better secondary stability (3) because high placement torques although they increase primary stability may are not favourable in the clinical setting (23). Bone remodelling was shown to counteract the OMI primary stability already 3 weeks after the OMI placement (15). In accordance to this, the removal torque exceeded the placement torque at the end of 10 months of orthodontic treatment (24).

The resistance to extraction of OMIs depends on various factors including OMI design, shear strength (secondary stability) and bone density. Migliorati and coworkers (25) have demonstrated that the thread depth and the pitch are also predictors for the load values in pull-out test. We suggest that the ideal thread depth to outer diameter ratio should be around $30 \%$ or less to avoid weakness of the shank (Nos 3,8). A sharp low thread angle is desired to reduce placement torque and risk of fracture (No 4). In case of dense bone, a cylindrical shape OMI or a less conical shape OMI with sharp threads is preferable to avoid excessive friction and compression. The 
conical Dual Top screw (No 8) although mechanically superior to other OMI types with respect to its high IT and stiffness had the greatest resistance to fracture in this group (Fig. 3) (26). Cylindrical OMIs showed no differences in POS values but significantly lower IT values compared to conical OMIs (Table 3 ).

-Large Diameter OMIs: Our data confirm that large diameter OMIs with conical design have higher IT values but provide lesser primary stability in terms of POS than large OMIs with a cylindrical design (11) (Table $3)$. An increases insertion torques are directly related to cortical bone thickness (27). However, very high placement torques will increase tensile and compressive stress to the peri-implant bone tissue, thus, being of disadvantage. The cylindrical OMI shape superiority was evident in the holding power. In general terms, the TF variable of the $2.0 \mathrm{~mm}$ OMIs is $60 \%$ higher compared to the median group.

OMIs with a diameter of $2 \mathrm{~mm}$ or more showed a 1.8fold lower risk of failing compared to OMIs with 1.2 $\mathrm{mm}$ diameter (28). Immediate or early loading up to $2 \mathrm{~N}$ had no impact on OMI stability (18).

The domaine of large and long diameter OMIs is the anchorage for molar distalizers or maxillary bone-born hybrid hyrax expanders (29) requiring higher loading forces. Long flutes along the axis and deep thread depth around $40 \%$ of such screws (No. 11) may increase the fracture risk (37.08 Ncm, the lowest of its group; Fig. 3). Short bowl flutes that store squeezed chips may, however, be of advantage due to a decrease in placement torque and increased bending strength.

In our study OMI No.10 fractured during insertion at a mean of about $44 \mathrm{~N}$ (depth $8 \mathrm{~mm}$ ). This is close to the observation by Mischkowski and co-workers (26). They observed head fractures of this OMI at $53-56 \mathrm{~N}$ at a depth of $10 \mathrm{~mm}$. It remains to be assessed why in the study by Wilmes and co-workers (17) these screws fractured within the force range of $23-41 \mathrm{~N}$ (total number of screws tested not stated).

\section{References}

1. Kong L, Hu K, Li D, Song Y, Yang J, Wu Z, et al. Evaluation of the cylinder implant thread height and width: a 3-dimensional finite element analysis. Int J Oral Maxillofac Implants. 2008;23:65-74.

2. Hong J, Lim YJ, Park SO. Quantitative biomechanical analysis of the influence of the cortical bone and implant length on primary stability. Clin Oral Implants Res. 2012;23:1193-7.

3. Wilmes B, Rademacher C, Olthoff G, Drescher D. Parameters affecting primary stability of orthodontic mini-implants. J Orofac Orthop. 2006;67:162-74.

4. Shah AH, Behrents RG, Kim KB, Kyung HM, Buschang PH. Effects of screw and host factors on insertion torque and pullout strength. Angle Orthod. 2012;82:603-10.

5. Chapman JR, Harrington RM, Lee KM, Anderson PA, Tencer AF, Kowalski D. Factors affecting the pullout strength of cancellous bone screws. J Biomech Eng. 1996;118:391-8.

6. Song YY, Cha JY, Hwang CJ. Mechanical specifications of various orthodontic mini-screws in relation to artificial cortical bone thickness. Angle Orthod. 2007;77:979-85.
7. Jolley T, Chung C. Peak torque values at fracture of orthodontic miniscrews. J Clin Orthod. 2007;41:326-8.

8. Kim YK, Kim YJ, Yun PY, Kim JW. Effects of the taper shape, dual-thread, and length on the mechanical properties of mini-implants. Angle Orthod. 2009;79:908-14.

9. Petrey JS, Saunders MM, Kluemper GT, Cunningham LL, Beeman CS. Temporary anchorage device insertion variables: effects on retention. Angle Orthod. 2010;80:446-53.

10. Barros SE, Janson G, Chiqueto K, Garib DG, Janson M. Effect of mini-implant diameter on fracture risk and self-drilling efficacy. Am J Orthod Dentofacial Orthop. 2011;140:e181-92.

11. Florvaag B, Kneuertz P, Lazar F, Koebke J, Zöller E, Braumann $\mathrm{B}$, et al. Biomechanical properties of orthodontic miniscrews. An invitro study. J Orofac Orthop. 2010;71:53-67.

12. Lee NK, Baek SH. Effects of the diameter and shape of orthodontic mini-implants on microdamage to the cortical bone. Am J Orthod Dentofacial Orthop. 2010;138:8.e1-8.

13. Cho KC, Baek SH. Effects of predrilling depth and implant shape on the mechanical properties of orthodontic mini-implants during the insertion procedure. Angle Orthod. 2012;82:618-24.

14. Rebaudi A, Laffi N, Benedicenti S, Angiero F, Romanos GE. Microcomputed tomographic analysis of bone reaction at insertion of orthodontic mini-implants in sheep. Int J Oral Maxillofac Implants. 2011;26:1233-40.

15. Cha JY, Takano-Yamamoto T, Hwang CJ. The effect of miniscrew taper morphology on insertion and removal torque in dogs. Int J Oral Maxillofac Implants. 2010;25:777-83.

16. Hong C, Lee H, Webster R, Kwak J, Wu BM, Moon W. Stability comparison between commercially available mini-implants and a novel design: part 1. Angle Orthod. 2011;81:692-9.

17. Wilmes B, Drescher D. Impact of bone quality, implant type, and implantation site preparation on insertion torques of mini-implants used for orthodontic anchorage. Int J Oral Maxillofac Surg. 2011;40:697-703.

18. Crismani AG, Bertl MH, Celar AG, Bantleon HP, Burstone CJ. Miniscrews in orthodontic treatment: review and analysis of published clinical trials. Am J Orthod Dentofacial Orthop. 2010;137:108-13.

19. Chen Y, Kyung HM, Gao L, Yu WJ, Bae EJ, Kim SM. Mechanical properties of self-drilling orthodontic micro-implants with different diameters. Angle Orthod. 2010;80:821-7.

20. Togni F, Baras F, Ribas Mde O, Taha MO. Histomorphometric analysis of bone tissue repair in rabbits after insertion of titanium screws under different torque. Acta Cir Bras. 2011;26:261-6.

21. Motoyoshi M, Hirabayashi M, Uemura M, Shimizu N. Recommended placement torque when tightening an orthodontic mini-implant. Clin Oral Implants Res. 2006;17:109-14.

22. Motoyoshi M, Yoshida T, Ono A, Shimizu N. Effect of cortical bone thickness and implant placement torque on stability of orthodontic mini-implants. Int J Oral Maxillofac Implants. 2007;22:779-84.

23. McManus MM, Qian F, Grosland NM, Marshall SD, Southard TE. Effect of miniscrew placement torque on resistance to miniscrew movement under load. Am J Orthod Dentofacial Orthop. 2011;140:e93-8.

24. Suzuki EY, Suzuki B. Placement and removal torque values of orthodontic miniscrew implants. Am J Orthod Dentofacial Orthop. 2011;139:669-78.

25. Migliorati M, Signori A, Silvestrini-Biavati A. Temporary anchorage device stability: an evaluation of thread shape factor. Eur J Orthod. 2012;34:582-6.

26. Mischkowski RA, Kneuertz P, Florvaag B, Lazar F, Koebke J, Zöller JE. Biomechanical comparison of four different miniscrew types for skeletal anchorage in the mandibulo-maxillary area. Int J Oral and Maxillofac Surg. 2008;37:948-54.

27. Pithon MM, Nojima MG, Nojima LI. In vitro evaluation of insertion and removal torques of orthodontic mini-implants. Int J Oral Maxillofac Surg. 2011;40:80-5.

28. Schätzle M, Männchen R, Zwahlen M, Lang NP. Survival and failure rates of orthodontic temporary anchorage devices: a systematic review. Clin Oral Implants Res. 2009;20:1351-9. 
29. Ludwig B, Glas B, Bowman SJ, Drescher D, Wilmes B. Miniscrew-supported Class III treatment with the Hybrid RPE Advancer. J Clin Orthod. 2010;44:533-9.

Acknowledgments

We would like to thank Sigrun Chrubasik / Freiburg for her advice during preparation of the manuscript and L Gil / Barcelona for his technical assistance. 